\title{
Sarcopenia-related features and factors associated with lower muscle strength and physical performance in older Chinese: a cross sectional study
}

Ping Zeng, Yiwen Han, Jing Pang, Sinan Wu, Huan Gong, Jianguo Zhu, Jian Li and Tiemei Zhang*

\begin{abstract}
Background: The associations of sarcopenia with adverse health status have highlighted the importance of sarcopenia research and intervention. This study was designed to analyze the characteristics of aging-related differences in appendicular skeletal muscle mass (ASM), handgrip strength (HS), gait speed (GS) and their associated factors in older Chinese, in order to generate guidance for sarcopenia intervention in this population.

Methods: Population-based cross-sectional study. The criteria proposed by Asian Working Group for Sarcopenia were used to define low ASM, HS, and GS. The time required for five repeated chair stands (RCS) was also measured to evaluate physical performance. The differences of continuous variables were compared using one-way ANOVA tests and the Pearson correlation was used to analyze the relationship of each measurement adjusted by gender and age. Stepwise logistic regression was used to determine associated factors of low HS and low physical performance.

Results: The data were analyzed in a total of 218 younger adults (aged 20-59, 76 males, 142 females) and 461 older adults ( $\geq 60$ year, 207 males and 254 females). There were significant differences among age groups for HS, GS, and RCS while females were found to have significantly lower HS and GS values. ASM was significantly correlated with HS but not with other measures. Correlations among HS and GS, RCS were influenced by age differences. In the older group, unstructured daily routine $(O R=2.77)$ was associated with the risk of low $G S$, while physical exercise $(O R=0.27)$, and engaging in hobbies $(O R=0.11)$ were associated with faster $G$. Co-morbidity $(\mathrm{OR}=1.99)$ was associated with the risk of reduced performance of RCS, while engaging in hobbies was associated with faster RCS performance $(\mathrm{OR}=0.35)$.

Conclusions: Muscle strength and physical performance varied with aging in older Chinese. Measures of GS, HS, and RCS provide a readily available and effective method for assessing the risk of functional mobility decline. Maintaining a healthy life style and physical activity throughout life is beneficial for older people to improve their physical performance, especially in the early stages of aging.
\end{abstract}

Keywords: Sarcopenia, Muscle mass, Muscle strength, Physical performance

\footnotetext{
* Correspondence: tmzhang126@126.com

The Key Laboratory of Geriatrics, Beijing Hospital \& Beijing Institute of Geriatrics, Ministry of Health, Beijing 100730, China
} 


\section{Background}

Sarcopenia has been widely recognized as a syndrome featured by age-related loss of skeletal muscle mass, decrease of muscle strength and/or physical performance in older populations $[1,2]$. Because of the associations of sarcopenia with adverse health status [3-8], it has become a subject of increased focus in geriatrics research. In 2014, the Asian Working Group for Sarcopenia (AWGS) has proposed a consensus for the diagnosis of sarcopenia [9]. Taking an approach similar to that of the European Working Group on Sarcopenia in Older Peoples (EWGSOP) [10], diagnostic criteria for three parameters (muscle mass, muscle strength, and physical performance) were determined. However, ethnic diversity in these measurements has been found [11-14], even within Asia [14]. Furthermore, the prevalence of reduced muscle mass reported in Asian populations is extremely low [15-17], while it is a pre-requisite for the diagnosing of sarcopenia. Except for identifying individuals at especially high risk of sarcopenia, the exact definition of sarcopenia may not be as important as developing criteria for intervention to maintain the function of older people. Some studies have confirmed the potential benefits of sarcopenia intervention approaches [18-21] in reversing age-related declines in muscle mass and physical performance while increasing in mortality, which support the importance of further understanding the sarcopenia-related factors in national populations, especially in China that has a large size of rapidly aging population and a huge number of disabled older people. In turn, this information can be used to formulate and implement strategies for sarcopenia intervention and prevention. In this paper, the age-related differences in muscle mass, muscle strength, and physical performance associated with sarcopenia are described. The correlations between each variable and the various factors associated with the parameters of sarcopenia are examined quantitatively.

\section{Methods}

\section{Study design}

This investigation used a cross-sectional study design. In response to the need for understanding the situation of sarcopenia in China, we collected data to examine agerelated variations of body muscle mass, muscle strength, and physical performance in two study and one reference groups. The survey was conducted during 2013 in two groups of older volunteers ( $\geq 60$ year), who were retired office workers living in the urban area of Beijing and older farmers living in Jingzhuang, Yanqing, 80 kilometer northwest of Beijing. In addition, young adult (20-59 years) employees of Beijing Hospital also volunteered to participate to make up the young reference group. Data were gathered from 465 older participants and 220 younger adults. Due to incomplete data collection or artificial implants, such as cardiac pacemakers or joints which hinder the subjects completing the bioimpedance measurement of body composition, the data were only analyzed for 679 subjects (76 young males, 142 young females, 207 older males and 254 older females).

Questionnaires and physical examinations were used to collect information on demographics, life style, history of diseases, physical status, activities of daily living (ADL), skeletal muscle mass, muscle strength, and physical performance. The life style variables included: current smoking ( $\geq 5 /$ day); current alcohol consumption (at least once a week); engaging in physical exercise (moderate or more, at least once a week and lasting for 30 min or more); meals per day; daily routine (daily time schedule, 1 = structured, 2 = moderate, 3 = unstructured); and having hobbies (playing chess, reading, drawing, painting, handwriting, singing, planting, fishing, keeping pets). Meals per day was investigated in this study as a surrogate of nutrition intake. Daily routine and hobbies were considered as they may indicate the psychological status and life attitude of older people. Individuals with 2 or more common diseases, such as hypertension, diabetes, cardiovascular diseases, coronary heart disease, chronic obstructive pulmonary disease (COPD), osteoarthritis, and cancer, were considered as having comorbidity. All the participants were ambulatory without major physical disabilities. The interviewers were trained before the survey was administered; the subjects' informed consent was obtained to use their information in this study; and the study was approved by the Ethics Committee of Beijing Hospital, Ministry of Health.

\section{Body composition measurements}

Subjects were dressed in light clothes and were barefoot. Weight and height were measured using a calibrated digital scale. Weights were rounded to the nearest $0.1 \mathrm{~kg}$, and heights were rounded to the nearest $0.1 \mathrm{~cm}$. Body mass index (BMI) was calculated by dividing the weight by the square of the height $\left(\mathrm{kg} / \mathrm{m}^{2}\right)$.

Body composition was measured with a body composition analyzer (BCA-2A, Tongfang Health Technology Company, Tshinghua, Beijing, China), which conducted a direct segmental multi-frequency ( $5 \mathrm{kHz}$ to $500 \mathrm{kHz}$ ) bioelectrical impedance analysis (BIA). The details of measurement of body mass by BCA-2A have been published elsewhere [22]. In short, the BCA-2A has eight tactile electrodes allowing the subjects' hands and feet to be separately measured for bioelectrical resistance. While being measured, the participants stood on the device in bare feet with legs slightly apart while holding the electrodes in both hands with arms away from the trunk. After the measurement, the analyzer generated skeletal muscle mass (SM), appendicular skeletal muscle mass (ASM), lean mass (LM), and fat mass (FM) reports 
on the screen. The measurements were calibrated using a patented formula obtained by magnetic resonance imaging (MRI) analysis from a group of healthy Chinese subjects. The ASM index (ASMI), SM index (SMI), and LM index (LMI) were obtained by dividing ASM, SM, and LM by the square of height, respectively $\left(\mathrm{kg} / \mathrm{m}^{2}\right)$.

\section{Muscle strength and physical performance measurements} Muscle strength was determined by handgrip strength (HS) and was measured in each hand using a hand dynamometer (WCS-II, Beijing). The grip strength was measured twice for each hand, and the higher of the two values was used in the analysis.

Two physical performance measures were considered in this study: usual gait speed (GS) and five times repeated chair stands (RCS). GS was evaluated by the $6-\mathrm{m}$ gait speed (GS) test. Participants walked at their usual pace from a standing start and continued walking to a point past the line indicating the end of the course. The total time-from the "start" command issued by the investigator to the first step over the end line-was measured with a stopwatch. GS was calculated by dividing distance in meters by the time in seconds $(\mathrm{m} / \mathrm{s})$. When measuring the time for RCS, participants were asked to rise from a sitting position and stand up completely as quickly as possible, repeating this movement five times in rapid succession, without using their arms. The exercise was stopped if there were concerns about the subject's safety or if the procedure could not be completed within 1 min.

\section{Definition of low muscle mass, muscle strength and physical performance}

The criteria proposed by the Asian Working Group for Sarcopenia (AWGS) were used to define sarcopenia related parameters, i.e. low muscle mass, low muscle strength, and low physical performance (defined by low GS). Reduced physical performance of RCSs means a longer time to complete the RCSs. Since the cut-off value to define reduced performance of RCS was not available in AWGS, the approach of previous work for generating a reference value was used [11,23]. That is, a reduced performance of RCS was defined as a value in excess of the 80th percentile of RCSs measured in the older participants in this study. As a result, RCS $\geq 12.5 \mathrm{~s}$ was defined as reduced RCS performance.

\section{Statistical analysis}

Descriptive statistics were used to characterize the demographics and measured variables of the subjects. The differences of continuous variables between younger and older adults were compared using one-way ANOVA tests. To analyze the degrees of differences in ASMI, GS, HS, and RCS among age groups, the mean values $\left(X_{n}\right)$ of the variables obtained in the groups aged $\geq 50(50-59$, $60-69,70-79,80+)$ were compared with those (X) obtained in the younger adults (20-49 years), and the degrees of difference were calculated as $100 *\left(\mathrm{X}_{\mathrm{n}}-\mathrm{X}\right) / \mathrm{X}$ (Comparison 1). The mean values $\left(\mathrm{X}_{\mathrm{n}}\right)$ of these variables obtained in the groups aged $\geq 50(50-59,60-69,70-79$, 80-) were also compared with those of the next younger group $\mathrm{X}_{\mathrm{n}-1}$ (group 50-59 was compared with group 20-49), and the degrees of difference were calculated as $100 *\left(X_{n}-X_{n-1}\right) / X_{n-1}$ (Comparison 2). Pearson correlation was used to analyze the relationship of each measurement adjusted by gender and age. Stepwise logistic regression was used to determine associated factors of low HS and low physical performance. The statistical analyses were carried out using SAS software licensed to the Chinese Center for Disease Control and Prevention, and $p<0.05$ was considered as statistically significant.

\section{Results \\ Descriptions of anthropometry, muscle strength and physical performance data between young and older groups}

The data concerning anthropometry, muscle strength, and physical performance of young group and older group are shown in Table 1. Older people had significant lower LM, ASM, HS, but higher fat mass, and slower GS and RCS than young people $(\mathrm{p}<0.0001)$. Males had significant higher LM, ASM, HS, and quicker GS, but lower FM than females $(\mathrm{p}<0.05)$. The gender difference for RCS was not significant.

\section{Degrees of difference in muscle mass, muscle strength, and physical performance by age and gender}

The degrees of difference in ASMI, GS, HS and RCS with age are shown in Table 2. Declines in ASMI, GS, and HS were observed after years of 60s. When the mean values of the measured variables obtained in the groups aged $\geq 50(50-59,60-69,70-79,80+)$ were compared with those of younger adults (20-49, Comparison 1), GS declined more in females (17.24-40.69\%) than in males (18.00-28.67\%), while HS declined more in males (9.56-34.58 \%) than in females (16.70$28.54 \%)$. The time required to complete RCS increased more dramatically and earlier (at 50) than did other variables, with $17.49-84.90 \%$ and $40.96-197.76 \%$ increases in male and females, respectively. When the mean values of the measured variables obtained in the groups aged $\geq$ $50(50-59,60-69,70-79,80-)$ were compared with those of the next younger group (Comparison 2), two obvious declines in each variable (RCS increase) were observed in 60- and 80-year old group (Table 2, Comparison 2). The decline of ASMI was less significant than other variables in both males and females. 
Table 1 The descriptions of anthropometry, muscle strength and physical performance data of young group and elderly group (mean \pm SD)

\begin{tabular}{|c|c|c|c|c|c|c|}
\hline \multirow[t]{2}{*}{ Characteristic } & \multicolumn{2}{|c|}{ Young group (20-59) } & \multicolumn{2}{|c|}{ Older group $(\geq 60)$} & \multirow[b]{2}{*}{ p1 } & \multirow[b]{2}{*}{$\mathrm{p} 2$} \\
\hline & Males $(n=76)$ & Female $(n=142)$ & Male $(n=207)$ & Female $(n=254)$ & & \\
\hline Average age (year) & $38.6 \pm 12.0$ & $41.7 \pm 12.6$ & $71.0 \pm 5.7$ & $69.2 \pm 6.5$ & $<0.0001$ & 0.6297 \\
\hline \multicolumn{7}{|l|}{ Anthropometric measurements } \\
\hline Height (m) & $1.73 \pm 0.07$ & $1.61 \pm 0.05$ & $1.67 \pm 0.06$ & $1.56 \pm 0.06$ & $<0.0001$ & $<0.0001$ \\
\hline Weight (kg) & $72.76 \pm 11.22$ & $59.55 \pm 8.90$ & $66.93 \pm 10.00$ & $60.05 \pm 9.97$ & 0.0221 & $<0.0001$ \\
\hline Fat mass (kg) & $14.84 \pm 4.99$ & $17.61 \pm 5.60$ & $16.48 \pm 5.47$ & $20.57 \pm 5.97$ & $<0.0001$ & $<0.0001$ \\
\hline Lean mass (kg) & $54.43 \pm 6.82$ & $39.13 \pm 4.10$ & $47.63 \pm 5.43$ & $37.39 \pm 4.88$ & $<0.0001$ & $<0.0001$ \\
\hline Arm skeletal mass (kg) & $7.90 \pm 1.08$ & $5.84 \pm 2.52$ & $6.88 \pm 0.81$ & $5.40 \pm 0.74$ & $<0.0001$ & $<0.0001$ \\
\hline Leg skeletal mass (kg) & $19.35 \pm 2.43$ & $13.90 \pm 1.46$ & $16.96 \pm 1.95$ & $13.30 \pm 1.73$ & $<0.0001$ & $<0.0001$ \\
\hline ASM (kg) & $27.25 \pm 3.44$ & $19.46 \pm 2.06$ & $23.84 \pm 2.75$ & $18.70 \pm 2.42$ & $<0.0001$ & $<0.0001$ \\
\hline Fat mass (\%) & $19.90 \pm 4.04$ & $28.98 \pm 5.42$ & $24.17 \pm 5.56$ & $33.73 \pm 5.73$ & $<0.0001$ & $<0.0001$ \\
\hline Lean mass (\%) & $75.00 \pm 3.76$ & $66.25 \pm 5.12$ & $71.66 \pm 5.89$ & $62.82 \pm 5.73$ & $<0.0001$ & $<0.0001$ \\
\hline Arm skeletal mass (\%) & $10.92 \pm 0.96$ & $9.91 \pm 4.64$ & $10.35 \pm 0.90$ & $9.08 \pm 1.00$ & 0.0001 & $<0.0001$ \\
\hline Leg skeletal mass (\%) & $26.72 \pm 1.32$ & $23.54 \pm 1.82$ & $25.52 \pm 2.09$ & $22.35 \pm 2.03$ & $<0.0001$ & $<0.0001$ \\
\hline Percentage of ASM (\%) & $37.55 \pm 2.12$ & $32.93 \pm 2.38$ & $35.87 \pm 2.98$ & $31.42 \pm 2.93$ & $<0.0001$ & $<0.0001$ \\
\hline $\mathrm{BMI}\left(\mathrm{kg} / \mathrm{m}^{2}\right)$ & $24.09 \pm 3.12$ & $22.87 \pm 3.30$ & $23.88 \pm 3.13$ & $24.59 \pm 3.71$ & 0.0005 & 0.6346 \\
\hline $\mathrm{LMI}\left(\mathrm{kg} / \mathrm{m}^{2}\right)$ & $17.97 \pm 1.62$ & $15.02 \pm 1.30$ & $16.99 \pm 1.45$ & $15.29 \pm 1.56$ & 0.1064 & $<0.0001$ \\
\hline Arm skeletal mass index $\left(\mathrm{kg} / \mathrm{m}^{2}\right)$ & $2.62 \pm 0.32$ & $2.24 \pm 0.98$ & $2.46 \pm 0.23$ & $2.21 \pm 0.24$ & 0.0357 & $<0.0001$ \\
\hline Leg skeletal mass index $\left(\mathrm{kg} / \mathrm{m}^{2}\right)$ & $6.42 \pm 0.61$ & $5.34 \pm 0.46$ & $6.05 \pm 0.52$ & $5.44 \pm 0.56$ & 0.0755 & $<0.0001$ \\
\hline $\operatorname{ASMI}\left(\mathrm{kg} / \mathrm{m}^{2}\right)$ & $9.00 \pm 0.85$ & $7.47 \pm 0.65$ & $8.50 \pm 0.74$ & $7.65 \pm 0.78$ & 0.2292 & $<0.0001$ \\
\hline \multicolumn{7}{|c|}{ Muscle strength and body physical function } \\
\hline Handgrip strength $(\mathrm{kg})$ & $40.81 \pm 5.77$ & $26.44 \pm 4.42$ & $34.04 \pm 8.05$ & $21.56 \pm 5.64$ & $<0.0001$ & $<0.0001$ \\
\hline Gait Speed (m/s) & $1.51 \pm 0.24$ & $1.45 \pm 0.22$ & $1.19 \pm 0.26$ & $1.15 \pm 0.31$ & $<0.0001$ & 0.0200 \\
\hline Chair repeat time (s) & $6.58 \pm 2.01$ & $6.66 \pm 4.27$ & $10.66 \pm 5.46$ & $11.42 \pm 7.41$ & $<0.0001$ & 0.2287 \\
\hline
\end{tabular}

p1: Age difference; p2: gender difference (across full sample)

Correlation among appendicular muscle mass, appendicular muscle mass index, muscle strength, and physical performance

The Pearson correlation coefficients among ASM, ASMI, HS, GS, and RCS are shown in Table 3. In the older groups, ASM associated with HS $(r=0.28$ in males, $p<$ $0.001 ; r=0.30$ in females, $p<0.001)$; GS correlated with HS $(r=0.35$ in males, $p<0.001 ; \mathrm{r}=0.40$ in females, $p<$ $0.001)$ and RCS ( $r=0.26$ in males, $p<0.001 ; r=0.23$ in females, $p<0.001$ ). In the adults (age $<60$ ), only appendicular muscle mass and HS were significantly associated (for ASM and HS, $r=0.24$ in males, $p<0.001 ; r=$ 0.35 in females, $p<0.001)$. The correlations of ASMI with other variables were very weak in both older and adults groups.

Factors associated with low muscle strength and reduced physical performance in the older group

Factors, such as age, gender, lifestyle, and health status associated with low muscle strength, reduced physical performance are shown in Table 4. In general, aging was associated with the risk of low muscle strength and reduced physical performance, and rural farmers were weaker in muscle strength and physical performance. $\mathrm{BMI}$ and unstructured daily routine were associated with the risk of low GS, with OR (95 \% CI): 1.25 (1.09-1.43) and 2.77(1.34-5.72), respectively, while physical exercise was associated with faster GS, having OR (95 \% CI): 0.27 (0.09-0.79). Engagement in hobbies was also associated with better performance in both GS and RCS, OR (95 \% CI): $0.11(0.04-0.33)$ and $0.35(0.17-0.72)$, respectively. In addition, co-morbidity was associated with the risk of reduced performance of RCS, OR (95\% CI): 1.99(1.20-3.29).

\section{Discussion}

In this study, patterns of differences in muscle mass, muscle strength, and physical performance over time, the correlation among these parameters, and other associated factors were analyzed in a Chinese population. The most obvious changes were observed in muscle strength and physical performance in the age 60-69 and 
Table 2 The degrees of difference in sarcopenia related parameters with age

\begin{tabular}{|c|c|c|c|c|c|c|c|c|c|c|c|c|c|}
\hline \multirow[b]{2}{*}{ Males } & \multirow[b]{2}{*}{$\mathrm{n}$} & \multicolumn{3}{|c|}{ ASM index $\left(\left(\mathrm{kg} / \mathrm{m}^{2}\right)\right.$} & \multicolumn{3}{|c|}{ Gait speed $(\mathrm{m} / \mathrm{s})$} & \multicolumn{3}{|c|}{ Handgrip strength (kg) } & \multicolumn{3}{|c|}{ Repeated chair stands (s) } \\
\hline & & Mean \pm SD & Comparison1 (\%) & Comparison2 (\%) & Mean \pm SD & Comparison1 (\%) & Comparison2 (\%) & Mean \pm SD & Comparison1 (\%) & Comparison2 (\%) & Mean \pm SD & Comparison1 (\%) & Comparison 2 (\%) \\
\hline $20-49$ & 56 & $8.88 \pm 0.84$ & - & - & $1.50 \pm 0.24$ & - & - & $40.6 \pm 5.8$ & - & - & $6.29 \pm 2.0$ & - & - \\
\hline $50-59$ & 20 & $9.31 \pm 0.84$ & 4.84 & 4.84 & $1.53 \pm 0.25$ & 2.00 & 2.00 & $41.53 \pm 5.73$ & 2.29 & 2.29 & $7.39 \pm 1.84$ & 17.49 & 17.49 \\
\hline $60-69$ & 82 & $8.55 \pm 0.73$ & -3.72 & -8.16 & $1.23 \pm 0.28$ & -18.00 & -19.61 & $36.72 \pm 8.26$ & -9.56 & -11.58 & $10.63 \pm 7.62$ & 69.00 & 43.84 \\
\hline $70-79$ & 112 & $8.50 \pm 0.68$ & -4.28 & -0.58 & $1.18 \pm 0.25$ & -21.33 & -4.07 & $33.37 \pm 7.45$ & -17.81 & -9.12 & $10.46 \pm 2.59$ & 66.30 & -1.60 \\
\hline $80-$ & 13 & $8.26 \pm 1.23$ & -6.98 & -2.82 & $1.07 \pm 0.29$ & -28.67 & -9.32 & $26.56 \pm 5.89$ & -34.58 & -20.41 & $11.63 \pm 3.65$ & 84.90 & 11.19 \\
\hline \multicolumn{14}{|c|}{ Females } \\
\hline $20-49$ & 92 & $7.31 \pm 0.53$ & - & - & $1.45 \pm 0.19$ & - & - & $26.1 \pm 3.9$ & - & - & $5.81 \pm 1.40$ & - & - \\
\hline $50-59$ & 50 & $7.69 \pm 0.84$ & 5.20 & 5.20 & $1.46 \pm 0.28$ & 0.69 & & $26.95 \pm 5.13$ & 3.26 & 3.26 & $8.19 \pm 6.63$ & 40.96 & 40.96 \\
\hline $60-69$ & 146 & $7.69 \pm 0.78$ & 5.20 & 0.00 & $1.20 \pm 0.29$ & -17.24 & -17.81 & $21.74 \pm 5.62$ & -16.70 & -19.33 & $10.17 \pm 3.32$ & 75.04 & 24.18 \\
\hline 70-79 & 86 & $7.66 \pm 0.78$ & 4.79 & -0.39 & $1.13 \pm 0.30$ & -22.07 & -5.83 & $21.54 \pm 5.59$ & -17.47 & -0.92 & $11.82 \pm 7.76$ & 103.44 & 16.22 \\
\hline 80- & 22 & $7.14 \pm 0.68$ & -2.33 & -6.79 & $0.86 \pm 0.22$ & -40.69 & -23.89 & $18.65 \pm 5.12$ & -28.54 & -13.42 & $17.30 \pm 16.46$ & 197.76 & 46.36 \\
\hline
\end{tabular}

Comparison 1, compare the mean values $\left(X_{n}\right)$ of the variable obtained in the groups aged $\geq 50$ with those $(X)$ obtained in the 20-49 years group, and calculated as $100^{*}\left(X_{n}-X\right) / X$; Comparison 2: compare the mean values $\left(X_{n}\right)$ of the variable obtained in the groups aged 50 with the mean values $X_{n-1}$ of the next younger group, and calculated as $100^{*}\left(X_{n}-X_{n-1}\right) / X_{n-1}$ 
Table 3 The correlation coefficient among appendicular muscle mass, appendicular muscle mass index, mass strength, gait speed, and repeated chair stands $(r)$

\begin{tabular}{lllllll}
\hline \multicolumn{3}{l}{$\geq 60$ years } & \multicolumn{5}{l}{$<60$ years } \\
\hline Male & HS & GS & RCS & HS & GS & RCS \\
ASM (kg) & $0.28^{*}$ & 0.02 & 0.06 & $0.24^{* *}$ & 0.01 & 0.01 \\
ASM index & 0.05 & -0.07 & 0.07 & $0.25^{* *}$ & -0.02 & 0.05 \\
HS & & $0.35^{*}$ & $-0.15^{* *}$ & & 0.17 & 0.04 \\
RCS & $-0.14^{* *}$ & $-0.26^{*}$ & & 0.04 & 0.08 & \\
Female & & & & & & \\
ASM (kg) & $0.30^{*}$ & 0.05 & -0.02 & 0.35 & 0.05 & -0.08 \\
ASM index & 0.07 & -0.11 & 0.03 & $0.30^{*}$ & -0.09 & 0.04 \\
HS & & $0.40^{*}$ & $-0.17^{*}$ & & 0.09 & -0.08 \\
RCS & $-0.17^{*}$ & $-0.23^{*}$ & & -0.08 & -0.12 & \\
\hline
\end{tabular}

*: $\mathrm{p}<0.01 ; * * \mathrm{p}<0.05$. ASM appendicular muscle mass, HS handgrip strength, $G S$ gait speed, $R C S$ repeated chair stands

$80+$ groups. Additionally, muscle strength and physical performance (indicated by GS) showed significant gender differences, with females having poorer muscle strength and lower GS (Table 2). Our data imply that before the age of 60 , there could be opportunities to intervene and prevent the decline of muscle mass or muscle physical function, and that older women should have greater concern about functional declines in their later years than men. An earlier study reported that a noticeable decrease in absolute skeletal muscle mass did not occur until the end of fifth decade life, which was attributed to a decrease in a lower body SM [24]. Our data show a very dramatic decline in RCS, which may represent the decline of leg muscle strength, supporting the idea that an important benefit may be gained by maintaining muscle mass and strength in the lower extremities.

Studies in China [25] and Asia [15, 16] have found a low prevalence of low ASM, especially among older women. In our previous report [22], several factors which may cause bias in the determination of the reference value of for ASM were discussed to explain this phenomenon. In this study, the prevalence of low ASM was only $1.44 \%$ in males and $0.39 \%$ in females. Since low ASM is a pre-requisite for the definition of sarcopenia, the prevalence of sarcopenia is expected to be low in these populations. Our results demonstrated that muscle strength and physical performance declined to a greater extent than did muscle mass, which is consistent with previous reports (Table 2) [26].

Statistical correlations among the sarcopenia parameters (e.g., ASM, HS, GS, and RCS) were analyzed to investigate associations among these factors. In our study, ASM was only correlated with HS (both in adult and older groups; Table 3), not with physical performance. Similar results were also reported in a Taiwanese population [13]. The correlations among muscle strength and physical performance were shown to vary by age category. In the adults, these correlations were not statistically significant $(p>0.05)$, while in the older group, GS was statistically correlated with HS and RCS. The age specific association of muscle function supports sarcopenia being described as part of the geriatric syndrome [27]. Some reports have demonstrated that HS and GS are associated with adverse health-related events in the older population $[4,28-30]$. The poor association between muscle mass and physical performance, and low prevalence of reduced muscle mass may indicate that,

Table 4 Factors associated with low handgrip strength, low gait speed and reduced performance in repeat chair stands (RCS) by stepwise logistic regression (OR: $95 \% \mathrm{Cl}$ )

\begin{tabular}{llll}
\hline Variables & Low handgrip strength & Low gait speed & Reduced performance of RCS \\
\hline Age increased 10 years & $1.13(1.07-1.18)$ & $1.12(1.04-1.18)$ & $1.10(1.06-1.15)$ \\
Urban resident & $0.10(0.05-0.20)$ & $0.08(0.02-0.40)$ & $0.39(0.24-0.65)$ \\
Female & $1.92(1.06-3.46)$ & - & - \\
BMI & - & $1.25(1.09-1.43)$ & - \\
$\geq 2$ co-morbidity & - & - & $-1.99(1.20-3.29)$ \\
Current smoker & - & - & - \\
Current Drinker & - & $0.27(0.09-0.79)$ & - \\
Physical exercise & - & - & - \\
Meals per day & - & $2.77(1.34-5.72))$ & $0.35(0.17-0.72)$ \\
Structured daily routine & - & $0.11(0.04-0.33)$ & - \\
Hobby loving & - & & \\
Self rated health & - & & -
\end{tabular}

Current smoker: $\geq 5 /$ day; Current drinker: at least once a week; Physical exercise: moderate or more, at least once a week and lasting for 30 min or more; Meals/day: $1=1$ meal, $2=2$ meals, $3 \geq 3$ meals; Structured daily routine: $1=$ structured, $2=$ moderate, $3=$ unstructured; Self rated health: $1=$ very bad, $2=$ bad, $3=$ modest, $4=$ good, $5=$ very good 
even without muscle mass measurement (which is still challenged by precision and proper definition), the measurement of muscle strength and physical performance would be good predictors of future functional status in the older population. These parameters are also easily obtained in screening or clinical tests to evaluate the muscle strength and physical performance in older people.

Using the AWGS criteria, $5.31 \%$ of males, and $7.50 \%$ of females had low GS; $13.10 \%$ of males and $22.86 \%$ of females had low HS in our older population. Although RCS was not a parameter proposed by AWGS for sarcopenia screening, other researchers have found RCS to be a sensitive indicator of the strength of the lower limbs [29] and is, therefore, included in our list of parameters. Our study found that the time to complete five RCS increased more dramatically and earlier (at 50s) than other variables, with $17.5-84.9 \%$ and $41.0-197.8 \%$ increases in male and females, respectively. Using the approach of previous work for generating reference values [11, 23], a value in the excess 80 th percentile of RCSs $(\geq 12.5 \mathrm{~s}$.) measured in the older people of this study was defined as the criterion for indicating reduced RCS performance. Using this criterion, $18.36 \%$ of older males and $27.60 \%$ of older females presented reduced RCS performance. Another study defined a cut-point of $17.05 \mathrm{~s}$ and showed that it was an adequate substitute to predict adverse health events [29]. In other studies, Caucasians were shown to have longer RCS than Asians [14, 31]. The ethnic differences in RCS may reflect the higher BMI and body weights of Caucasians.

Lifestyle factors, such as nutrition, physical activity, exercise, alcohol intake, and tobacco use, have an impact on the occurrence of sarcopenia [32-34]. Our study demonstrated that physical performances are more likely to be correlated with lifestyle factors, while HS is not. Greater strength may be more associated with a better nutrition, especially nutrition experienced in the early stages of life or even during fetal development [35, 36]. This observation leads to the special concerns about the nutrition status of older Chinese, since nutrition is associated with muscle mass, and our population has a relatively higher prevalence of reduced HS (13.10\% in males and $22.86 \%$ in females). Obesity and unstructured daily schedule were associated with the risk of low GS, while physical exercise is associated with faster GS. Engagement in hobbies was associated with better performance in both GS and RCS. These results may indicate that a positive and healthy life style contributes to improved physical performance of the older people [37]. In addition, among three parameters; i.e., HS, GS, and RCS, co-morbidity was the only factor associated with the risk of reduced performance of RCS. Whether RCS contains more information about the health status in the older population needs further study, our investigation supports the importance of measuring RCS to evaluate lower extremity functions in the older people.

This study has several limitations. First, it is a crosssectional study, which makes it less likely to observe the changes of muscle mass, muscle strength, and physical performance with aging. Specifically, the study group was relatively healthy volunteers, and the sample size was not sufficiently large; therefore, it is difficult to produce a representative description of sarcopenia in our population. However, at a time when related studies are still scarce, our study provides information that is helpful to form potential strategies for early intervention and prevention of sarcopenia in older Chinese. Also, due to the low prevalence of reduced muscle mass in this population, the specific factors associated with sarcopenia cannot be evaluated. However, potential factors that may influence muscle strength and physical performance have been identified and significant associations have been observed.

\section{Conclusions}

Physical performance and muscle strength declined with age in an older Chinese population. Measures of physical performance and muscle strength are easily obtained and represent an effective way to assess the risk of mobility functional decline in the older people. Maintaining a healthful life style and physical activity throughout the lifecourse is beneficial for older people to improve their physical performance, especially in the early stage of aging.

\section{Abbreviations}

ASM: appendicular skeletal muscle mass; ASMI: appendicular skeletal mass index; AWGS: the Asian Working Group for Sarcopenia; BIA: bioelectrical impedance analyzer; BMI: body mass index; EWGSOP: the European Working Group on Sarcopenia in Older Peoples; GS: gait speed; HS: handgrip strength: LM: lean mass; MRI: magnetic resonance imaging; RCS: repeated chair stands; SM: skeletal muscle mass.

\section{Competing interest}

The authors declare that they have no competing interests.

\section{Authors' contributions}

$\mathrm{PZ}$ designed the study, conducted the analyses, and wrote the manuscript. YWH, JP, SNW, HG and JGZ made contributions to the conception and design of the study, the acquisition of data and review the manuscript. JL and TMZ contributed to the design of the study, interpretation of data, and revising the article critically for important intellectual content. All authors read and approved the final manuscript.

\section{Acknowledgements}

This study was funded by National Department Public Benefit Research Foundation by Ministry of Health P. R. China (No. 201302008). Yi Zhang, Enyi Zhang, Yan Zhang, Yuan Miao and Jing Yang involved in the survey and data entry portions of this study. We would like to express our appreciation to Dr. Donald Barnes, South China University of Technology, for helpful comments on the final revision of this paper. We also would like to say thanks to Dr. Robert Lynch, College of Public Health, the University of Oklahoma Health Sciences Center, for his instructive comments on our revised version of this manuscript.

Received: 23 August 2015 Accepted: 4 February 2016

Published online: 15 February 2016 


\section{References}

1. Rosenberg $\mathbb{H}$. Sarcopenia: origins and clinical relevance. J Nutr. 1997;127:990 S-91S.

2. Delmonico MJ, Harris TB, Visser M, Park SW, Conroy MB, Velasquez-Mieyer P, et al. Longitudinal study of muscle strength, quality, and adipose tissue infiltration. Am J Clin Nutr. 2009;90:1579-85.

3. Cesari M, Pahor M, Lauretani F, Zamboni V, Bandinelli S, Bernabei R, et al. Skeletal muscle and mortality results from the InCHIANTI Study. J Gerontol A Biol Sci Med Sci. 2009:64:377-84.

4. Newman AB, Kupelian V, Visser M, Simonsick EM, Goodpaster BH, Kritchevsky SB, et al. Strength, but not muscle mass, is associated with mortality in the health, aging and body composition study cohort. J Gerontol A Biol Sci Med Sci. 2006;61:72-7.

5. Arango-Lopera VE, Arroyo P, Gutiérrez-Robledo LM, Perez-Zepeda MU, Cesari M. Mortality as an adverse outcome of sarcopenia. J Nutr Health Aging. 2013;17:259-62.

6. Tanimoto $Y$, Watanabe M, Sun W, Tanimoto K, Shishikura K, Sugiura Y, et al. Association of sarcopenia with functional decline in community-dwelling elderly subjects in Japan. Geriatr Gerontol Int. 2013. doi:10.1111/ggi.12037.

7. Goodpaster BH, Park SW, Harris TB, Kritchevsky SB, Nevitt M, Schwartz AV, et al. The loss of skeletal muscle strength, mass, and quality in older adults: the health, aging and body composition study. J Gerontol A Biol Sci Med Sci. 2006;61:1059-64

8. Janssen I, Shepard DS, Katzmarzyk PT, Roubenoff R. The healthcare costs of sarcopenia in the United States. J Am Geriatr Soc. 2004;52:80-5.

9. Chen LK, Liu LK, Woo J, Assantachai P, Auyeung TW, Bahyah KS, et al. Sarcopenia in Asia: consensus report of the Asian Working Group for Sarcopenia. JAMDA. 2014;15:95-101.

10. Cruz-Jentoft AJ, Baeyens JP, Bauer JM, Boirie Y, Cederholm T, Landi F, et al. Sarcopenia: European consensus on definition and diagnosis: Report of the European Working Group on Sarcopenia in Older People. Age Ageing. 2010;39:412-23

11. Newman AB, Kupelian V, Visser M, Simonsick E, Goodpaster B, Nevitt M, et al. Sarcopenia: alternative definitions and associations with lower extremity function. J Am Geriatr Soc. 2003;51:1602-9.

12. Coin A, Sarti S, Ruggiero E, Giannini S, Fedrazzoni M, Minisola S. Prevalence of sarcopenia based on different diagnostic criteria using DEXA and appendicular skeletal muscle mass reference values in an Italian population aged 20 to 80. J Am Med Dir Assoc. 2013;14:507-12.

13. Liu LK, Lee WJ, Liu CL, Chen LY, Lin MH, Peng LN, et al. Age-related skeletal muscle mass loss and physical performance in Taiwan: Implications to diagnostic strategy of sarcopenia in Asia. Geriatr Gerontol Int. 2013. doi:10.1111/ggi.12040.

14. Woo J, Arai H, Ng TP, Sayer AA, Wong M, Syddall H, et al. Ethnic and geographic variations in muscle mass, muscle strength and physical performance measures. Eur Geriatr Med. 2014;5:155-64.

15. Kim YS, Lee Y, Chung YS, Lee D, Joo NS, Hong D, et al. Prevalence of sarcopenia and sarcopenic obesity in the Korean population based on the Fourth Korean National Health and Nutritional Examination Surveys. J Gerontol A Biol Sci Med Sci. 2012;67:1107-13.

16. Lau EM, Lynn HS, Woo JW, Kwok TC, Melton 3rd LJ. Prevalence of and risk factors for sarcopenia in elderly Chinese men and women. J Gerontol A Biol Sci Med Sci. 2005;60:213-6.

17. Lee WJ, Liu LK, Peng LN, Lin MH, Chen LK. Comparisons of sarcopenia defined by IWGS and EWGSOP criteria among older people: results from the I-Lan longitudinal aging study. J Am Med Dir Assoc. 2013;14:528 e1-7.

18. Harber MP, Konopka AR, Douglass MD, Minchev K, Kaminsky LA, Trappe TA, et al. Aerobic exercise training improves whole muscle and single myofiber size and function in older women. Am J Physiol Regul Integr Comp Physiol. 2009;297:R1452-9.

19. McCartney N, Hicks AL, Martin J, Martin CE. Long-term resistance training in the elderly: effects on dynamic strength, exercise capacity, muscle, and bone. J Gerontol A Biol Sci Med Sci. 1995:50:B97-104.

20. Liu CJ, Latham NK. Progressive resistance strength training for improving physical function in older adults. Cochrane Database Syst Rev. 2009;2009, CD002759.

21. Schlicht J, Camaione DN, Owen SV. Effect of intense strength training on standing balance, walking speed, and sit-to-stand performance in older adults. J Gerontol A Biol Sci Med Sci. 2001;56:M281-6.

22. Zeng P, Wu S, Han Y, Liu J, Zhang Y, Zhang E, et al. Differences in body composition and physical functions associated with sarcopenia in Chinese elderly: Reference values and prevalence. Arch Gerontol Geriatr. 2015;60:118-23.
23. Fried LP, Tangen CM, Walston J, Newman AB, Hirsch C, Gottdiener J, et al. Frailty in older adults: evidence for a phenotype. J Gerontol A Biol Sci Med Sci. 2001;56:M146-57.

24. Janssen I, Heymsfield SB, Wang ZM, Ross R. Skeletal muscle mass and distribution in 468 men and women aged $18-88$ years. J Appl Physiol. 2000;89:81-8.

25. Wen $X$, Wang $M$, Jiang $C M$, Zhang $Y M$. Are current definitions of sarcopenia applicable for older Chinese adults? J Nutr Health Aging. 2011;15:847-51.

26. Ferrucci L, Guralnik JM, Buchner D, Kasper J, Lamb SE, Simonsick EM, et al. Departures from linearity in the relationship between measures of muscular strength and physical performance of the lower extremities: the Women's Health and Aging Study. J Gerontol A Biol Sci Med Sci. 1997;52:M275-85.

27. Cruz-Jentoft AJ, Landi F, Topinková E, Michel JP. Understanding sarcopenia as a geriatric syndrome. Curr Opin Clin Nutr Metab Care. 2010;13:1-7.

28. Gale CR, Martyn CN, Cooper C, Sayer AA. Grip strength, body composition, and mortality. Int J Epidemiol. 2007;36:228-35. doi:10.1093/ije/dy1224.

29. Cesari M, Kritchevsky SB, Newman AB, Simonsick EM, Harris TB, Penninx BW et al. Added value of physical performance measures in predicting adverse health-related events: results from the health, aging and body composition study. J Am Geriatr Soc. 2009;57:251-9.

30. Taekema DG, Gussekloo J, Maier AB, Westendorp RG, de Craen AJ. Handgrip strength as a predictor of functional, psychological and social health-A prospective population-based study among the oldest old. Age Ageing. 2010;39:331-7.

31. Patel HP, Syddall HE, Jameson K, Robinson S, Denision H, Roberts HC, et al. Prevalence of sarcopenia in community-dwelling older people in the UK using the European Working Group on Sarcopenia in Older People (EWGSOP) definition: findings from the Hertfordshire Cohort Study (HCS). Age Ageing. 2013;42:378-84

32. Burton LA, Sumukadas D. Optimal management of sarcopenia. Clin Interv Aging. 2010;5:217-28.

33. Visvanathan R, Chapman I. Preventing sarcopenia in older people. Maturitas. 2010;66:383-8.

34. Rom O, Kaisari S, Aizenbud D, Reznick AZ. Identification of possible cigarette smoke constituents responsible for muscle catabolism. J Muscle Res Cell Motil. 2012;33:199-208

35. Kuh D, Bassey J, Hardy R, Aihie SA, Wadsworth M, Cooper C. Birth weight childhood size, and muscle strength in adult life: evidence from a birth cohort study. Am J Epidemiol. 2002;156:627-33.

36. Sayer AA, Syddall HE, Gilbody HJ, Dennison EM, Cooper C. Does sarcopenia originate in early life? Findings from the Hertfordshire cohort study. J Gerontol A Biol Sci Med Sci. 2004:59:M930-4.

37. Freiberger E, Sieber C, Pfeifer K. Physical activity, exercise, and sarcopenia_future challenges. Wien Med Wochenschr. 2011;161:416-25.

\section{Submit your next manuscript to BioMed Central and we will help you at every step:}

- We accept pre-submission inquiries

- Our selector tool helps you to find the most relevant journal

- We provide round the clock customer support

- Convenient online submission

- Thorough peer review

- Inclusion in PubMed and all major indexing services

- Maximum visibility for your research

Submit your manuscript at www biomedcentral com/submit
C BioMed Central 